\title{
A product of invariant random permutations has the same small cycle structure as uniform*
}

\author{
Mohamed Slim Kammoun ${ }^{\dagger} \quad$ Mylène Maïda ${ }^{\ddagger}$
}

\begin{abstract}
We use moment method to understand the cycle structure of the composition of two independent invariant permutations. We prove that under a good control on fixed points and cycles of length 2 , the limiting joint distribution of the number of small cycles is the same as in the uniform case i.e. for any positive integer $k$, the number of cycles of length $k$ converges to the Poisson distribution with parameter $\frac{1}{k}$ and is asymptotically independent of the number of cycles of length $k^{\prime} \neq k$.
\end{abstract}

Keywords: random permutations; moment method; universality results. MSC2020 subject classifications: 60C05; 60B20; 60F05; 05A16; 05A05.

Submitted to ECP on October 21, 2019, final version accepted on July 5, 2020.

Supersedes arXiv: 1910.04031.

Supersedes HAL : hal - 02309521.

\section{Introduction and main results}

We denote by $\mathfrak{S}_{n}$ the group of permutations of $\{1, \ldots, n\}$, by $\#_{k} \sigma$ the number of cycles of $\sigma$ of length $k$, by $\# \sigma$ the total number of cycles of $\sigma$ and by $\operatorname{tr}(\sigma):=\#_{1} \sigma$.

The cycle structure of a permutation chosen uniformly among the symmetric group $\mathfrak{S}_{n}$ is well understood (see e.g. [2] for detailed results). In particular, the following classical result holds:

Theorem 1.1. [1, Theorem 3.1] If $\sigma_{n}$ follows the uniform distribution on $\mathfrak{S}_{n}$ then for any $k \geq 1$,

$$
\left(\#_{1} \sigma_{n}, \ldots, \#_{k} \sigma_{n}\right) \underset{n \rightarrow \infty}{\stackrel{d}{\longrightarrow}} \eta_{k}:=\left(\xi_{1}, \xi_{2}, \ldots, \xi_{k}\right)
$$

where $\underset{n \rightarrow \infty}{\stackrel{d}{\longrightarrow}}$ denotes the convergence in distribution, $\xi_{1}, \xi_{2}, \ldots, \xi_{k}$ are independent and the distribution of $\xi_{d}$ is Poisson of parameter $\frac{1}{d}$.

In this work, we question the universality class of this convergence. We show that a product of conjugation invariant permutations that do not have too many fixed points and cycles of size 2 lies within this class. More precisely, we have the following.

\footnotetext{
*This work is partially supported by the Labex CEMPI (ANR-11-LABX-0007-01).

${ }^{\dagger}$ Univ. Lille, CNRS, UMR 8524 - Laboratoire Paul Painlevé, F-59000 Lille, France.

E-mail: mohamed-slim. kammoun@univ-lille. $\mathrm{fr}$

${ }^{\ddagger}$ Univ. Lille, CNRS, UMR 8524 - Laboratoire Paul Painlevé, F-59000 Lille, France.

E-mail: mylene.maida@univ-lille.fr
} 
A product of invariant random permutations has the same small cycle structure

Theorem 1.2. Let $\left(\sigma_{n}\right)_{n \geq 1}$ and $\left(\rho_{n}\right)_{n \geq 1}$ be two sequences of random permutations such that for any $n \geq 1, \sigma_{n} \in \mathfrak{S}_{n}$ and $\rho_{n} \in \mathfrak{S}_{n}$. For any $k \geq 1$, let $t_{k}^{n}:=\#_{k}\left(\sigma_{n} \rho_{n}\right)$. Assume that

- For any $n \geq 1, \sigma_{n}$ and $\rho_{n}$ are independent.

- For any $n \geq 1$, for any $\sigma \in \mathfrak{S}_{n}, \sigma^{-1} \sigma_{n} \sigma \stackrel{d}{=} \sigma_{n}$, or $\sigma^{-1} \rho_{n} \sigma \stackrel{d}{=} \rho_{n}$.

- For any $k \geq 1$,

$$
\begin{gathered}
\lim _{n \rightarrow \infty} \mathbb{E}\left(\left(\frac{\#_{1} \sigma_{n}}{\sqrt{n}}\right)^{k}\right)=0 \quad \text { and } \quad \lim _{n \rightarrow \infty} \mathbb{E}\left(\left(\frac{\#_{1} \rho_{n}}{\sqrt{n}}\right)^{k}\right)=0, \\
\lim _{n \rightarrow \infty} \frac{\mathbb{E}\left(\#_{2} \sigma_{n}\right)}{n}=0 \quad \text { and } \quad \lim _{n \rightarrow \infty} \frac{\mathbb{E}\left(\#_{2} \rho_{n}\right)}{n}=0 .
\end{gathered}
$$

Then for any $k \geq 1$,

$$
\left(t_{1}^{n}, t_{2}^{n}, \ldots, t_{k}^{n}\right) \underset{n \rightarrow \infty}{\stackrel{d}{\longrightarrow}} \eta_{k} .
$$

The result can be extended to more than two permutations. We refer the reader to Section 3.1 for details. Similar results have been obtained in [9] for permutations that are equicontinuous in both coordinates and converging as a permuton (see definitions there). With the motivation of random gluing of polygons, a stronger convergence (in total variation distance) was established in [4] when one of the permutations has all its cycles of length at least 3 (see also [5]) and in [3] when one of the permutations is an involution without fixed point. None of the previous results covers for example the product of two Ewens distributions. They are known to satisfy the convergences required in $H_{3}$ and $H_{4}$ so that our result tells that the product of two Ewens distributions behaves like a uniform permutation, as far as small cycles are concerned. In our framework, in the case of two permutations, a weaker result can be obtained without any hypothesis on the cycles of size 2 .

Proposition 1.3. With the same notations as in Theorem 1.2, under $H_{1}, H_{2}$ and $H_{3}$, we have convergence of the first moment i.e for any $e \geq 1$,

$$
\lim _{n \rightarrow \infty} \mathbb{E}\left(t_{e}^{n}\right)=\frac{1}{e}
$$

Note that when one of the permutations $\sigma_{n}$ or $\rho_{n}$ follows the uniform distribution, under $H_{1}$, the product also follows the uniform distribution and Theorem 1.2 is a direct consequence of Theorem 1.1.

Our motivation to understand the cycle structure of random permutations is the relation, in the case of conjugation invariant permutations, to the longest common subsequence (LCS) of two permutations. For example, using [6, Theorem 1.2] and that $L C S(\sigma, \rho)$ is equal to the length of the longest increasing subsequence of $\sigma^{-1} \rho$, if $\sigma_{n}^{-1} \rho_{n}$ is conjugation invariant and

$$
\frac{\#\left(\sigma_{n}^{-1} \rho_{n}\right)}{\sqrt[6]{n}} \underset{n \rightarrow \infty}{\stackrel{d}{\longrightarrow}} 0,
$$

then for any $s \in \mathbb{R}$,

$$
\mathbb{P}\left(\frac{L C S\left(\sigma_{n}, \rho_{n}\right)-2 \sqrt{n}}{\sqrt[6]{n}} \leq s\right) \underset{n \rightarrow \infty}{\longrightarrow} F_{2}(s),
$$

where $F_{2}$ is the cumulative distribution function of the GUE Tracy-Widom distribution.

Another motivation comes from traffic distributions, a non-commutative probability theory introduced in [8] to understand the moments of permutation invariant random matrices. As shown in [8], the limit in traffic distribution of uniform permutation matrices 
A product of invariant random permutations has the same small cycle structure

is trivial but Theorem 1.1 can be seen as a second-order result in this framework. It is therefore natural to ask about limiting joint fluctuations for the product of several permutation matrices, which is a really non-commutative case. To emphasize this relation, we rewrite Theorem 1.2 as follows.

Corollary 1.4. Under $H_{1}, H_{2}, H_{3}$ and $H_{4}$, for any $k \geq 1,\left(\operatorname{tr}(\sigma \rho), \operatorname{tr}\left((\sigma \rho)^{2}\right), \ldots, \operatorname{tr}\left((\sigma \rho)^{k}\right)\right)$ converges in distribution to $\left(\xi_{1}, \xi_{1}+2 \xi_{2}, \ldots, \sum_{d \mid k} d \xi_{d}\right)$, where $\xi_{1}, \xi_{2}, \ldots$ are independent and the distribution of $\xi_{d}$ is Poisson of parameter $\frac{1}{d}$.

It means that in this framework, the fluctuations are only governed by an hypothesis on fixed points and cycles of order two. The optimality of conditions $H_{3}$ and $H_{4}$ will be discussed at the end of the paper.

\section{Proof of results}

We begin with a few preliminary remarks and simplifications.

First of all, the equivalence between Theorem 1.2 and Corollary 1.4 is due to the following classical argument. For any $\sigma \in \mathfrak{S}_{n}$, if $c_{i}(\sigma)$ denotes the length of the cycle of $\sigma$ containing $i$,

$$
\operatorname{tr}\left(\sigma^{k}\right)=\sum_{i=1}^{n} \mathbb{1}_{\sigma^{k}(i)=i}=\sum_{i=1}^{n} \mathbb{1}_{c_{i}(\sigma) \mid k}=\sum_{j \mid k} \sum_{i=1}^{n} \mathbb{1}_{c_{i}(\sigma)=j}=\sum_{j \mid k} j \#_{j} \sigma .
$$

In the hypothesis $H_{2}$, we assume that one of the permutations, say $\sigma_{n}$, may not have a conjugation invariant distribution. In fact, it is enough to prove Theorem 1.2 in the case where both permutations are conjugation invariant. Indeed, if we choose $\tau_{n}$ uniform and independent of the $\sigma$-algebra generated by $\sigma_{n}$ and $\rho_{n}$, the cycle structure of $\sigma_{n} \rho_{n}$ is the same as

$$
\tau_{n}^{-1} \sigma_{n} \rho_{n} \tau_{n}=\left(\tau_{n}^{-1} \sigma_{n} \tau_{n}\right)\left(\tau_{n}^{-1} \rho_{n} \tau_{n}\right) \stackrel{d}{=}\left(\tau_{n}^{-1} \sigma_{n} \tau_{n}\right) \rho_{n}
$$

and $\left(\tau_{n}^{-1} \sigma_{n} \tau_{n}\right)$ is also conjugation invariant.

\subsection{Preliminary results}

To prove Theorem 1.2, we will use the same objects introduced in [7, pages 12-13] where one can get further details and examples. To a couple of permutations and a subset of $p$ indices, we will associate a set of $2 p$ graphs. For technical reasons, we prefer working with $\sigma_{n}^{-1} \rho_{n}$ rather than $\sigma_{n} \rho_{n}$ : for any $k \geq 1$, we define $\tilde{t}_{k}^{n}:=\#_{k}\left(\sigma_{n}^{-1} \rho_{n}\right)$. Under $H_{2}, \sigma_{n} \stackrel{d}{=} \sigma_{n}^{-1}$ and consequently under $H_{1}$ and $H_{2}, \forall k \geq 1\left(t_{1}^{n}, t_{2}^{n}, \ldots, t_{k}^{n}\right)$ and $\left(\tilde{t}_{1}^{n}, \tilde{t}_{2}^{n}, \ldots, \tilde{t}_{k}^{n}\right)$ have the same distribution.

Let us now recall the combinatorial objects we will use.

- We denote by $\mathbb{G}_{k}^{n}$ the set of oriented simple graphs with vertices $\{1,2, \ldots, n\}$ and having exactly $k$ edges. We allow here loops but not multiple edges. Given $g \in \mathbb{G}_{k}^{n}$, we denote by $E_{g}$ the set of its edges and by $A_{g}:=\left[\mathbb{1}_{(i, j) \in E_{g}}\right]_{1 \leq i, j \leq n}$ its adjacency matrix.

- A connected component of $g$ is called trivial if it does not have any edge and a vertex $i$ of $g$ is called isolated if $E_{g}$ does not contain any edge of the form $(i, j)$ or $(j, i)$ nor a loop $(i, i)$. Let $g \in \mathbb{G}_{k}^{n}$, we denote by $\tilde{g}$ the graph obtained from $g$ after removing isolated vertices.

- We say that two oriented simple graphs $g_{1}$ and $g_{2}$ are isomorphic if one can obtain $g_{2}$ by changing the labels of the vertices of $g_{1}$. In particular, if $g_{1}, g_{2} \in \mathbb{G}_{k}^{n}$ then $g_{1}, g_{2}$ are isomorphic if and only if there exists a permutation matrix $\sigma$ such that $A_{g_{1}} \sigma=\sigma A_{g_{2}}$. 
A product of invariant random permutations has the same small cycle structure

- Let $\mathcal{R}$ be the equivalence relation such that $g_{1} \mathcal{R} g_{2}$ if $\tilde{g}_{1}$ and $\tilde{g}_{2}$ are isomorphic. We denote by $\widehat{\mathbb{G}}_{k}:=\cup_{n \geq 1} \mathbb{G}_{k}^{n} / \mathcal{R}$ the set of equivalence classes of $\cup_{n \geq 1} \mathbb{G}_{k}^{n}$ for the relation $\mathcal{R}$.

Let $n \in \mathbb{N}^{*}$ and $\sigma, \rho \in \mathfrak{S}_{n}$. Let $m \in\{1, \ldots, n\}$ be fixed.

- We denote by $\left(i_{1}^{m}(\sigma, \rho)=m, i_{2}^{m}(\sigma, \rho), \ldots, i_{k_{m}(\sigma, \rho)}^{m}(\sigma, \rho)\right)$ the cycle of $\sigma^{-1} \circ \rho$ containing $m$, so that $k_{m}(\sigma, \rho):=c_{m}\left(\sigma^{-1} \circ \rho\right)$ is the length of this cycle. For $l \leq k_{m}(\sigma, \rho)$, we define $j_{l}^{m}(\sigma, \rho):=\rho\left(i_{l}^{m}(\sigma, \rho)\right)$. In particular, $i_{1}^{m}(\sigma, \rho), i_{2}^{m}(\sigma, \rho), \ldots, i_{k_{m}(\sigma, \rho)}^{m}(\sigma, \rho)$ are pairwise distinct and $j_{1}^{m}(\sigma, \rho), j_{2}^{m}(\sigma, \rho), \ldots, j_{k_{m}}^{m}(\sigma, \rho)(\sigma, \rho)$ are pairwise distinct. For sake of simplicity, when it is clear, we will use the notations $k_{m}, i_{l}^{m}$ and $j_{l}^{m}$ instead of $k_{m}(\sigma, \rho), i_{l}^{m}(\sigma, \rho)$ and $j_{l}^{m}(\sigma, \rho)$ respectively.

- We denote by $\mathcal{G}_{1}^{m}(\sigma, \rho) \in \mathbb{G}_{k_{m}}^{n}$ and $\mathcal{G}_{2}^{m}(\sigma, \rho) \in \mathbb{G}_{k_{m}}^{n}$ the graphs with vertices $\{1, \ldots, n\}$ such that

$$
E_{\mathcal{G}_{1}^{m}(\sigma, \rho)}=\left\{\left(i_{1}^{m}, j_{k_{m}}^{m}\right)\right\} \bigcup\left(\bigcup_{l=1}^{k_{m}-1}\left\{\left(i_{l+1}^{m}, j_{l}^{m}\right)\right\}\right) \quad \text { and } \quad E_{\mathcal{G}_{2}^{m}(\sigma, \rho)}=\bigcup_{l=1}^{k_{m}}\left\{\left(i_{l}^{m}, j_{l}^{m}\right)\right\}
$$

and by $g_{\sigma}$ the graph such that $A_{g_{\sigma}}=\sigma$. By construction, for any positive integer $m \leq n, \mathcal{G}_{1}^{m}(\sigma, \rho)$ (resp. $\mathcal{G}_{2}^{m}(\sigma, \rho)$ ) is a sub-graph of $g_{\sigma}$ (resp. $g_{\rho}$ ). Moreover, we want to emphasize that $\mathcal{G}_{1}^{m}(\sigma, \rho)$ and $\mathcal{G}_{2}^{m}(\sigma, \rho)$ have the same set of non-isolated vertices. For $i \in\{1,2\}$, let $\widehat{\mathcal{G}}_{i}^{m}(\sigma, \rho)$ be the equivalence class of $\mathcal{G}_{i}^{m}(\sigma, \rho)$.

- Let $I=\left(s_{1}, s_{2}, \ldots, s_{l}\right)$ a set of distinct indices of $\{1, \ldots, n\}$. We denote by

$$
\mathcal{G}^{I}(\sigma, \rho)=\left(\mathcal{G}_{1}^{s_{1}}(\sigma, \rho), \mathcal{G}_{2}^{s_{1}}(\sigma, \rho), \mathcal{G}_{1}^{s_{2}}(\sigma, \rho), \ldots, \mathcal{G}_{1}^{s_{l}}(\sigma, \rho), \mathcal{G}_{2}^{s_{l}}(\sigma, \rho)\right)
$$

and

$$
\widehat{\mathcal{G}}^{I}(\sigma, \rho)=\left(\widehat{\mathcal{G}}_{1}^{s_{1}}(\sigma, \rho), \widehat{\mathcal{G}}_{2}^{s_{1}}(\sigma, \rho), \widehat{\mathcal{G}}_{1}^{s_{2}}(\sigma, \rho), \ldots, \widehat{\mathcal{G}}_{1}^{s_{l}}(\sigma, \rho), \widehat{\mathcal{G}}_{2}^{s_{l}}(\sigma, \rho)\right) .
$$

- For $i \in\{1,2\}$, let $\mathcal{G}_{i}^{\{1,2, \ldots, \ell\}}(\sigma, \rho)$ be the graph such that $E_{\mathcal{G}_{i}^{\{1,2, \ldots, \ell\}}(\sigma, \rho)}=\cup_{j=1}^{\ell} E_{\mathcal{G}_{i}^{j}(\sigma, \rho)}$ and $\widehat{\mathcal{G}}_{i}^{\{1,2, \ldots, \ell\}}(\sigma, \rho)$ be the equivalence class of $\mathcal{G}_{i}^{\{1,2, \ldots, \ell\}}(\sigma, \rho)$.

Using the conjugation invariance, Theorem 1.2 is a direct consequence of the following: under the same hypotheses, for any $\ell \geq 1$, for any $e_{1}, e_{2} \ldots, e_{\ell} \geq 1$,

$$
\lim _{n \rightarrow \infty} \sum_{\hat{g}_{i}, \hat{g}_{i}^{\prime} \in \widehat{\mathbb{G}}_{e_{i}}, 1 \leq i \leq \ell} n^{\ell} \mathbb{P}\left(\widehat{\mathcal{G}}^{\{1,2, \ldots, \ell\}}\left(\sigma_{n}, \rho_{n}\right)=\left(\hat{g}_{1}, \hat{g}_{1}^{\prime}, \hat{g}_{2}, \ldots \hat{g}_{\ell}^{\prime}\right)\right)=C_{e_{1}, e_{2}, \ldots, e_{\ell}},
$$

where $C_{e_{1}, e_{2}, \ldots, e_{\ell}}$ is a constant independent of the laws of the permutations. Note that, for any $e_{i} \geq 1$, the cardinal of $\widehat{\mathbb{G}}_{e_{i}}$ and therefore the number of terms of the sum is finite.

Let us explain briefly why $(*)$ implies Theorem 1.2 . First, for any $e_{1}, \ldots, e_{\ell}$, we have

$$
\begin{aligned}
\mathbb{P}\left(c_{1}\left(\sigma_{n}^{-1} \rho_{n}\right)=e_{1}, \ldots, c_{\ell}\left(\sigma_{n}^{-1} \rho_{n}\right)=e_{\ell}\right) & \\
= & \sum_{\hat{g}_{i}, \hat{g}_{i}^{\prime} \in \widehat{\mathbb{G}}_{e_{i}}, 1 \leq i \leq \ell} \mathbb{P}\left(\widehat{\mathcal{G}}^{\{1,2, \ldots, \ell\}}\left(\sigma_{n}, \rho_{n}\right)=\left(\hat{g}_{1}, \hat{g}_{1}^{\prime}, \hat{g}_{2}, \ldots \hat{g}_{\ell}^{\prime}\right)\right) .
\end{aligned}
$$

Moreover, using the relation (2.1), one can check that the joint moments of $\left(\tilde{t}_{1}^{n}, \ldots, \tilde{t}_{k}^{n}\right)$ can be expressed as follows: for any $\ell_{1}, \ldots, \ell_{m}$ in $\{1, \ldots, k\}$,

$$
\begin{aligned}
\mathbb{E}\left(\tilde{t}_{\ell_{1}}^{n}, \ldots \tilde{t}_{\ell_{m}}^{n}\right) & =\frac{1}{\ell_{1} \ldots \ell_{m}} \mathbb{E}\left(\prod_{p=1}^{m} \sum_{i_{p}=1}^{n} \mathbb{1}_{c_{i_{p}}\left(\sigma_{n}^{-1} \rho_{n}\right)=\ell_{p}}\right) \\
& =\frac{1}{\ell_{1} \ldots \ell_{m}} \sum_{\mathbf{i} \in\{1, \ldots, n\}^{m}} \mathbb{P}\left(c_{i_{1}}\left(\sigma_{n}^{-1} \rho_{n}\right)=\ell_{1}, \ldots, c_{i_{m}}\left(\sigma_{n}^{-1} \rho_{n}\right)=\ell_{m}\right) .
\end{aligned}
$$


A product of invariant random permutations has the same small cycle structure

For $\mathbf{i}:=\left(i_{1}, \ldots, i_{m}\right) \in\{1, \ldots, n\}^{m}$, denote $\operatorname{ker}(\mathbf{i})$ the partition of $\{1, \ldots, m\}$ such that $p$ and $q$ are in the same block whenever $i_{p}=i_{q}$. By conjugation invariance, for any $\mathbf{i} \in\{1, \ldots, n\}^{m}$, the quantity $\mathbb{P}\left(c_{i_{1}}\left(\sigma_{n}^{-1} \rho_{n}\right)=\ell_{1}, \ldots, c_{i_{m}}\left(\sigma_{n}^{-1} \rho_{n}\right)=\ell_{m}\right)$ depends only on $\operatorname{ker}(\mathbf{i})$ and is denoted $\mathbb{P}(\operatorname{ker}(\mathbf{i}))$. For any partition $\lambda$ of $\{1, \ldots, m\}$, if $|\lambda|$ denotes the number of blocks of a partition $\lambda$, there exist $e_{1}, \ldots, e_{|\lambda|}$ such that

$$
\mathbb{P}(\lambda)=\mathbb{P}\left(c_{1}\left(\sigma_{n}^{-1} \rho_{n}\right)=e_{1}, \ldots, c_{|\lambda|}\left(\sigma_{n}^{-1} \rho_{n}\right)=e_{|\lambda|}\right),
$$

so that

$$
\mathbb{E}\left(\tilde{t}_{\ell_{1}}^{n}, \ldots \tilde{t}_{\ell_{m}}^{n}\right)=\frac{1}{\ell_{1} \ldots \ell_{m}} \sum_{\lambda} n(n-1) \ldots(n-|\lambda|+1) \mathbb{P}(\lambda),
$$

which together with (2.2) makes the link between $\left(^{*}\right)$ and Theorem 2.

Before getting into the proof of $(*)$, let us gather some useful combinatorial and then probabilistic results.

Lemma 2.1. [7, Lemma 15] If $m_{1} \in\left\{i_{l}^{m_{2}}, 1 \leq l \leq k_{m_{2}}\right\}$, then $\mathcal{G}_{1}^{m_{1}}(\sigma, \rho)=\mathcal{G}_{1}^{m_{2}}(\sigma, \rho)$ and $\mathcal{G}_{2}^{m_{1}}(\sigma, \rho)=\mathcal{G}_{2}^{m_{2}}(\sigma, \rho)$.

Lemma 2.2. For any $m \leq n$, for any permutation $\sigma, \rho \in \mathfrak{S}_{n}$,

$$
A_{\mathcal{G}_{1}^{m}(\sigma, \rho)}=A_{\mathcal{G}_{2}^{\rho(m)}\left(\rho^{-1}, \sigma^{-1}\right)}^{T} .
$$

Proof. It is easy to see that by definition $k_{m}(\rho, \sigma)=k_{m}(\sigma, \rho)$ and

$$
\begin{aligned}
& j_{\ell}^{m}(\rho, \sigma)=j_{k_{m}(\sigma, \rho)-\ell+1}^{m}(\sigma, \rho), \forall 1 \leq \ell \leq k_{m}(\sigma, \rho), \\
& i_{\ell}^{m}(\rho, \sigma)=i_{k_{m}(\sigma, \rho)-\ell+2}^{m}(\sigma, \rho), \forall 2 \leq \ell \leq k_{m}(\sigma, \rho), \\
& i_{1}^{m}(\rho, \sigma)=i_{1}^{m}(\sigma, \rho)=m,
\end{aligned}
$$

and Lemma 2.2 follows

Lemma 2.3. If all non trivial connected components of $\mathcal{G}_{1}^{m_{1}}(\sigma, \rho)$ and $\mathcal{G}_{2}^{m_{1}}(\sigma, \rho)$ have 2 vertices then both $\mathcal{G}_{1}^{m_{1}}(\sigma, \rho)$ and $\mathcal{G}_{2}^{m_{1}}(\sigma, \rho)$ have no 2-cycles.

Proof. Using the symmetries of the problem (Lemmas 2.1 and 2.2), it suffices to prove that if all non trivial connected components of $\mathcal{G}_{1}^{1}(\sigma, \rho)$ and $\mathcal{G}_{2}^{1}(\sigma, \rho)$ have 2 vertices then it is impossible to have at the same time $(1,2) \in \mathcal{G}_{2}^{1}(\sigma, \rho)$ and $(2,1) \in \mathcal{G}_{2}^{1}(\sigma, \rho)$. To simplify notations, let $k_{1}:=k_{1}(\sigma, \rho)=c_{1}\left(\sigma^{-1} \circ \rho\right), i_{o}^{1}:=i_{o}^{1}(\sigma, \rho)$ and $j_{o}^{1}:=j_{o}^{1}(\sigma, \rho)$.

Let $A=\left\{\eta>1 ; j_{\eta}^{1} \in\left\{i_{1}^{1}, i_{2}^{1}, \ldots, i_{\eta-1}^{1}\right\}\right.$ or $\left.i_{\eta}^{1} \in\left\{j_{1}^{1}, j_{2}^{1}, \ldots, j_{\eta-1}^{1}\right\}\right\}$. Suppose that $(1,2) \in$ $\mathcal{G}_{2}^{1}(\sigma, \rho)$ and $(2,1) \in \mathcal{G}_{2}^{1}(\sigma, \rho)$ then $k_{1} \geq 2$ and there exists a unique $1<l \leq k_{1}$ such that $i_{l}^{1}=2$ and $j_{l}^{1}=1$ so that $A$ is non-empty. Let $\ell^{\prime}:=\inf (A) \geq 2$. Assume that $\ell^{\prime}>2$. If $j_{\ell^{\prime}}^{1} \in$ $\left\{i_{1}^{1}, i_{2}^{1}, \ldots, i_{\ell^{\prime}-1}^{1}\right\}$, then there exists $\ell^{\prime \prime}<\ell^{\prime}$ such that $j_{\ell^{\prime}}^{1}=i_{\ell^{\prime \prime}}^{1}$ and since the component of $\mathcal{G}_{2}^{1}(\sigma, \rho)$ containing $i_{\ell^{\prime}}^{1}$ has two vertices and by definition $\left(i_{\ell^{\prime}}^{1}, j_{\ell^{\prime}}^{1}\right)$ and $\left(i_{\ell^{\prime \prime}}^{1}, j_{\ell^{\prime \prime}}^{1}\right)$ are two edges of $\mathcal{G}_{2}^{1}(\sigma, \rho)$, then $j_{\ell^{\prime \prime}}^{1}=i_{\ell^{\prime}}^{1}$. Since $\left(i_{\ell^{\prime}}^{1}, j_{\ell^{\prime}-1}^{1}\right)=\left(j_{\ell^{\prime \prime}}^{1}, j_{\ell^{\prime}-1}^{1}\right)$ and $\left(i_{\ell^{\prime \prime}+1}^{1}, j_{\ell^{\prime \prime}}^{1}\right)$ are edges of $\mathcal{G}_{1}^{1}(\sigma, \rho)$ and since $\mathcal{G}_{1}^{1}(\sigma, \rho)$ has only connected components of size 2 , we have necessarily $i_{\ell^{\prime \prime}+1}^{1}=j_{\ell^{\prime}-1}^{1}$. One can check easily that $\ell^{\prime \prime}<\ell^{\prime}-2$ otherwise either $\mathcal{G}_{1}^{1}(\sigma, \rho)$ or $\mathcal{G}_{2}^{1}(\sigma, \rho)$ has a loop. Indeed, if $\ell^{\prime \prime}=\ell^{\prime}-2$, then $\left(i_{\ell^{\prime \prime}+1}^{1}, j_{\ell^{\prime \prime}+1}^{1}\right)=\left(j_{\ell^{\prime}-1}^{1}, j_{\ell^{\prime \prime}+1}^{1}\right)=\left(j_{\ell^{\prime}-1}^{1}, j_{\ell^{\prime}-1}^{1}\right)$ is an edge of $\mathcal{G}_{2}^{1}(\sigma, \rho)$ and if $\ell^{\prime \prime}=\ell^{\prime}-1$, then $\left(i_{\ell^{\prime \prime}+1}^{1}, j_{\ell^{\prime \prime}}^{1}\right)=\left(j_{\ell^{\prime}-1}^{1}, j_{\ell^{\prime \prime}}^{1}\right)=\left(j_{\ell^{\prime}-1}^{1}, j_{\ell^{\prime}-1}^{1}\right)$ is an edge of $\mathcal{G}_{1}^{1}(\sigma, \rho)$. This implies that $\ell^{\prime}-1 \in A$, which is absurd. $i_{\ell^{\prime}}^{1} \in\left\{j_{1}^{1}, j_{2}^{1}, \ldots, j_{\ell^{\prime}-1}^{1}\right\}$ can be treated using the same techniques and one can extend easily to $\ell^{\prime}=2$.

We now introduce the following notation: given $g \in \mathbb{G}_{k}^{n}$, we denote by

$$
\mathfrak{S}_{n, g}:=\left\{\sigma \in \mathfrak{S}_{n} ; \forall(i, j) \in E_{g}, \sigma(i)=j\right\} .
$$

In other words, $\mathfrak{S}_{n, g}$ is the set of permutations $\sigma$ such that $g$ is a sub-graph of $g_{\sigma}$. It is not difficult to prove the two following lemmas. 
A product of invariant random permutations has the same small cycle structure

Lemma 2.4. Let $g_{1}, g_{1}^{\prime}, g_{2}, \ldots, g_{k}^{\prime} \in \cup_{\ell} \mathbb{G}_{\ell}^{n}$ and let $g, g^{\prime}$ be such that $E_{g}=\cup_{\ell=1}^{k} E_{g_{i}}$ and $E_{g^{\prime}}=\cup_{\ell=1}^{k} E_{g_{i}^{\prime}}$. Assume that there exists $\rho, \sigma$ such that

$$
\mathcal{G}^{\{1,2, \ldots, k\}}(\sigma, \rho)=\left(g_{1}, g_{1}^{\prime}, g_{2}, \ldots, g_{k}^{\prime}\right)
$$

Then for any random permutations $\rho_{n}, \sigma_{n}$,

$$
\begin{aligned}
\mathbb{P}\left(\bigcap_{i=1}^{k}\left\{\sigma_{n} \in \mathfrak{S}_{n, g_{i}}, \rho_{n} \in \mathfrak{S}_{n, g_{i}^{\prime}}\right\}\right) & =\mathbb{P}\left(\mathcal{G}^{\{1,2, \ldots, k\}}\left(\sigma_{n}, \rho_{n}\right)=\left(g_{1}, g_{1}^{\prime}, g_{2}, \ldots, g_{k}^{\prime}\right)\right) \\
& =\mathbb{P}\left(\mathcal{G}_{1}^{\{1,2, \ldots, k\}}\left(\sigma_{n}, \rho_{n}\right)=g, \mathcal{G}_{2}^{\{1,2, \ldots, k\}}\left(\sigma_{n}, \rho_{n}\right)=g^{\prime}\right) .
\end{aligned}
$$

Proof. We will only prove the first equality. The second one can be obtained using the same argument.

Let $\sigma^{\prime}, \rho^{\prime}$ be two permutations. We have seen that $\mathcal{G}_{2}^{m}\left(\sigma^{\prime}, \rho^{\prime}\right)$ is a subset of $g_{\rho^{\prime}}$, so that

$$
\mathcal{G}_{2}^{m}\left(\sigma^{\prime}, \rho^{\prime}\right)=g_{m}^{\prime} \Rightarrow \rho^{\prime} \in \mathfrak{S}_{n, g_{m}^{\prime}}
$$

and that $\mathcal{G}_{1}^{m}\left(\sigma^{\prime}, \rho^{\prime}\right)$ is a subset of $g_{\sigma^{\prime}}$, so that

$$
\mathcal{G}_{1}^{m}\left(\sigma^{\prime}, \rho^{\prime}\right)=g_{m} \Rightarrow \sigma^{\prime} \in \mathfrak{S}_{n, g_{m}}
$$

Consequently,

$$
\mathbb{P}\left(\mathcal{G}^{\{1,2, \ldots, k\}}\left(\sigma_{n}, \rho_{n}\right)=\left(g_{1}, g_{1}^{\prime}, g_{2}, \ldots, g_{k}^{\prime}\right)\right) \leq \mathbb{P}\left(\bigcap_{i=1}^{k}\left\{\sigma_{n} \in \mathfrak{S}_{n, g_{i}}, \rho_{n} \in \mathfrak{S}_{n, g_{i}^{\prime}}\right\}\right)
$$

Now suppose that there exists $\rho^{\prime}, \sigma^{\prime}$ such that

$$
\mathcal{G}^{\{1,2, \ldots, k\}}\left(\sigma^{\prime}, \rho^{\prime}\right)=\left(g_{1}, g_{1}^{\prime}, g_{2}, \ldots, g_{k}^{\prime}\right)
$$

Let $\sigma, \rho$ such that $\sigma \in \cap_{i=1}^{k} \mathfrak{S}_{n, g_{i}}$ and $\rho \in \cap_{i=1}^{k} \mathfrak{S}_{n, g_{i}^{\prime}}$. By definition and by iteration on $\ell$, one can check that for any $\ell^{\prime} \leq k, i_{\ell^{\prime}}^{\ell}\left(\sigma^{\prime}, \rho^{\prime}\right)=i_{\ell^{\prime}}^{\ell}(\sigma, \rho)$ and $j_{\ell^{\prime}}^{\ell}\left(\sigma^{\prime}, \rho^{\prime}\right)=j_{\ell^{\prime}}^{\ell}(\sigma, \rho)$. Consequently,

$$
\mathcal{G}^{\{1,2, \ldots, k\}}(\sigma, \rho)=\left(g_{1}, g_{1}^{\prime}, g_{2}, \ldots, g_{k}^{\prime}\right) .
$$

Finally we obtain

$$
\mathbb{P}\left(\mathcal{G}^{\{1,2, \ldots, k\}}\left(\sigma_{n}, \rho_{n}\right)=\left(g_{1}, g_{1}^{\prime}, g_{2}, \ldots, g_{k}^{\prime}\right)\right) \geq \mathbb{P}\left(\bigcap_{i=1}^{k}\left\{\sigma_{n} \in \mathfrak{S}_{n, g_{i}}, \rho_{n} \in \mathfrak{S}_{n, g_{i}^{\prime}}\right\}\right)
$$

Lemma 2.5. [7, Lemma 16] Let $g_{1}, g_{2} \in \mathbb{G}_{k}^{n}$. Assume that there exists $\rho \in \mathfrak{S}_{n}$ such that $A_{g_{2}} \rho=\rho A_{g_{1}}$. If $\rho$ has a fixed point on any non-trivial connected component of $g_{1}$, then $\mathfrak{S}_{n, g_{1}} \cap \mathfrak{S}_{n, g_{2}}=\emptyset$ or $A_{g_{1}}=A_{g_{2}}$.

Lemma 2.6. For any graph $g \in \mathbb{G}_{k}^{n}$ having $f$ loops, $p$ non-trivial connected components and $v$ non-isolated vertices, for any random permutation $\sigma_{n}$ with conjugation invariant distribution on $\mathfrak{S}_{n}$,

$$
\mathbb{P}\left(\sigma_{n} \in \mathfrak{S}_{n, g}\right) \leq \frac{\mathbb{P}\left(\sigma_{n}(1)=1, \ldots, \sigma_{n}(f)=f\right)}{\frac{(n-p) !}{(n-v) !}} \leq \frac{(n-v) !}{(n-p) !} .
$$


A product of invariant random permutations has the same small cycle structure

Proof. It is an adaptation of the proof of [7, Corollary 17]. By conjugation invariance, one can suppose without loss of generality that the loops of $g$ are $(1,1),(2,2), \ldots(f, f)$ and the set of non-isolated vertices of $g$ are $\{1,2, \ldots, v\}$.

If there exist $i, j, l$, with $j \neq l$ such that $\{(i, j) \cup(i, l)\} \subset E_{g}$ or $\{(j, i) \cup(l, i)\} \subset E_{g}$ then $\mathfrak{S}_{n, g}=\emptyset$. Therefore, if $\mathfrak{S}_{n, g} \neq \emptyset$, then non-trivial connected components of $g$ having $w$ vertices are either cycles of length $w$ or isomorphic to $\bar{g}_{w}$, where $A_{\bar{g}_{w}}=\left[\mathbb{1}_{j=i+1}\right]_{1 \leq i, j \leq w}$.

Let $g \in \mathbb{G}_{k}^{n}$ such that $\mathfrak{S}_{n, g} \neq \emptyset$. Fix $p$ vertices $x_{1}=1, x_{2}=2, \ldots, x_{f}=f, x_{f+1}, \ldots, x_{p}$ each belonging to a different non-trivial connected components of $g$. Let $x_{p+1}<x_{p+2}<$ $\cdots<x_{v}$ be such that $\left\{x_{p+1}, \ldots, x_{v}\right\}=\{1,2, \ldots, v\} \backslash\left\{x_{1}, \ldots x_{p}\right\}$ be the other non-isolated vertices. Let

$$
F=\left\{\left(y_{i}\right)_{p+1 \leq i \leq v} ; y_{i} \in\{1,2, \ldots, n\} \backslash\left\{x_{1}, \ldots x_{p}\right\} \text { pairwise distinct }\right\} .
$$

Given $y=\left(y_{i}\right)_{p+1 \leq i \leq v} \in F$, we denote by $g_{y} \in \mathbb{G}_{k}^{n}$ the graph isomorphic to $g$ obtained by fixing the labels of $x_{1}, x_{2}, \ldots, x_{p}$ and by changing the labels of $x_{i}$ by $y_{i}$ for $p+1 \leq i \leq v$. Since non trivial connected components of $g$ of length $w$ are either cycles or isomorphic to $\bar{g}_{w}$, if $y \neq y^{\prime} \in F$, then $g_{y} \neq g_{y^{\prime}}$ and by Lemma 2.5, $\mathfrak{S}_{n, g_{y}} \cap \mathfrak{S}_{n, g_{y^{\prime}}}=\emptyset$. Since $\sigma_{n}$ is conjugation invariant, we have $\mathbb{P}\left(\sigma_{n} \in \mathfrak{S}_{n, g_{y}}\right)=\mathbb{P}\left(\sigma_{n} \in \mathfrak{S}_{n, g_{y^{\prime}}}\right)=\mathbb{P}\left(\sigma_{n} \in \mathfrak{S}_{n, g}\right)$. Remark also that for any $y \in F$ and any $i \leq f,(i, i)$ is a loop of $g_{y}$. Thus, $\mathfrak{S}_{n, g_{y}} \subset\left\{\sigma \in \mathfrak{S}_{n} ; \forall i \leq\right.$ $\left.f, \sigma_{n}(i)=i\right\}$ and thus

$$
\begin{aligned}
\mathbb{P}\left(\sigma_{n} \in \mathfrak{S}_{n, g}\right)=\frac{\sum_{y \in F} \mathbb{P}\left(\sigma_{n} \in \mathfrak{S}_{n, g_{y}}\right)}{\operatorname{card}(F)} & =\frac{\mathbb{P}\left(\sigma_{n} \in \cup_{y \in F} \mathfrak{S}_{n, g_{y}}\right)}{\operatorname{card}(F)} \\
& \leq \frac{\mathbb{P}\left(\sigma_{n}(1)=1, \ldots, \sigma_{n}(f)=f\right)}{\frac{(n-p) !}{(n-v) !}} \leq \frac{(n-v) !}{(n-p) !} .
\end{aligned}
$$

Lemma 2.7. Let $\sigma_{n}$ be a random permutation with conjugation invariant distribution on $\mathfrak{S}_{n}$ such that, for any $k \geq 1, \lim _{n \rightarrow \infty} \mathbb{E}\left(\left(\frac{\#_{1} \sigma_{n}}{\sqrt{n}}\right)^{k}\right)=0$. Then, for any $f \geq 1$,

$$
\mathbb{P}\left(\sigma_{n}(1)=1, \ldots, \sigma_{n}(f)=f\right)=o\left(n^{-\frac{f}{2}}\right) .
$$

Lemma 2.8. For any $p \geq 1$, let $g$ be a graph with $p$ non trivial components each having 2 vertices. Assume that at least one of these components is a cycle. Then for any random permutation $\sigma_{n}$ with conjugation invariant distribution on $\mathfrak{S}_{n}$,

$$
\mathbb{P}\left(\sigma_{n} \in \mathfrak{S}_{n, g}\right) \leq \frac{(n-2 p) ! \mathbb{P}\left(c_{1}\left(\sigma_{n}\right)=2\right)}{(n-p) !} .
$$

Proof. Remark that by conjugation invariance, one can suppose without loss of generality that the set of non-isolated vertices of $g$ are $\{1,2, \ldots, 2 p\}$ and that $(1,2),(2,1) \in E_{g}$. Using the same definitions as the previous proof with $f=0$ and $v=2 p$ and by choosing $x_{1}=1$, we have $\mathfrak{S}_{n, g_{y}} \subset\left\{\sigma \in \mathfrak{S}_{n} ; c_{1}(\sigma)=2\right\}$. Thus,

$$
\begin{aligned}
\mathbb{P}\left(\sigma_{n} \in \mathfrak{S}_{n, g}\right)=\frac{\sum_{y \in F} \mathbb{P}\left(\sigma_{n} \in \mathfrak{S}_{n, g_{y}}\right)}{\operatorname{card}(F)}=\frac{\mathbb{P}\left(\sigma_{n} \in \cup_{y \in F} \mathfrak{S}_{n, g_{y}}\right)}{\operatorname{card}(F)} & \leq \frac{\mathbb{P}\left(c_{1}\left(\sigma_{n}\right)=2\right)}{\operatorname{card}(F)} \\
& =\frac{(n-2 p) ! \mathbb{P}\left(c_{1}\left(\sigma_{n}\right)=2\right)}{(n-p) !} .
\end{aligned}
$$

By the previous combinatorial lemmas, we get that the main contribution will come from the following subset of graphs. Let $\mathcal{T}_{k}^{n} \subset \mathbb{G}_{k}^{n}$ be the set of graphs $g$ having exactly $k$ non trivial components each having one edge and two vertices. 
A product of invariant random permutations has the same small cycle structure

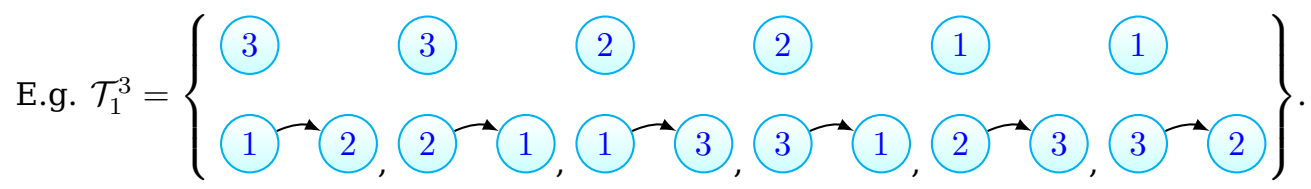

Let $\widehat{\mathcal{T}}_{k}$ be the equivalence class of the graphs of $\cup_{n} \mathcal{T}_{k}^{n}$. Their contribution is as follows.

Lemma 2.9. For any $p \geq 1, n \geq 2 p$ and any graph $g \in \mathcal{T}_{p}^{n}$, for any random permutation $\sigma_{n}$ with conjugation invariant distribution on $\mathfrak{S}_{n}$,

$$
\frac{(n-2 p) !}{(n-p) !}\left(1-\frac{p^{2}-p}{n-1}-p \mathbb{P}\left(\sigma_{n}(1)=1\right)\right) \leq \mathbb{P}\left(\sigma_{n} \in \mathfrak{S}_{n, g}\right) \leq \frac{(n-2 p) !}{(n-p) !} .
$$

Proof. The upper bound is due to Lemma 2.6 with $v=2 p$. Using the conjugation invariance again, one can suppose without loss of generality that

$$
E_{g}=\left\{\left(1, i_{1}\right),\left(2, i_{2}\right), \ldots,\left(p, i_{p}\right)\right\}
$$

where $i_{j}>p$ are all distinct. Let

$$
\mathfrak{S}_{n}^{p}=\left\{\sigma \in \mathfrak{S}_{n}, \forall i \leq p, \sigma(i)>p\right\}
$$

By conjugation invariance, for any $i \leq p$,

$$
\begin{aligned}
\mathbb{P}\left(\sigma_{n}(i) \leq p\right) & =\mathbb{P}\left(\sigma_{n}(1) \leq p\right)=\mathbb{P}\left(\sigma_{n}(1)=1\right)+\sum_{i=2}^{p} \mathbb{P}\left(\sigma_{n}(1)=i\right) \\
& =\mathbb{P}\left(\sigma_{n}(1)=1\right)+(p-1) \mathbb{P}\left(\sigma_{n}(1)=2\right) \\
& =\mathbb{P}\left(\sigma_{n}(1)=1\right)+(p-1) \frac{\sum_{i=2}^{n} \mathbb{P}\left(\sigma_{n}(1)=i\right)}{n-1} \\
& =\mathbb{P}\left(\sigma_{n}(1)=1\right)+\frac{\left(1-\mathbb{P}\left(\sigma_{n}(1)=1\right)\right)(p-1)}{n-1}
\end{aligned}
$$

Again by the conjugation invariance, we have that

$$
\begin{aligned}
\mathbb{P}\left(\sigma_{n} \in \mathfrak{S}_{n}^{p}\right)=1-\mathbb{P}\left(\sigma_{n} \in \mathfrak{S}_{n} \backslash \mathfrak{S}_{n}^{p}\right) & \geq 1-\sum_{i=1}^{p} \mathbb{P}\left(\sigma_{n}(i) \leq p\right) \\
& =1-p\left[\mathbb{P}\left(\sigma_{n}(1)=1\right)+\frac{\left(1-\mathbb{P}\left(\sigma_{n}(1)=1\right)\right)(p-1)}{n-1}\right] \\
& \geq 1-\frac{p^{2}-p}{n-1}-p \mathbb{P}\left(\sigma_{n}(1)=1\right) .
\end{aligned}
$$

In particular, if $\mathbb{P}\left(\sigma_{n} \in \mathfrak{S}_{n}^{p}\right)=0$, the lower bound is trivial and one can now assume that $\mathbb{P}\left(\sigma_{n} \in \mathfrak{S}_{n}^{p}\right)>0$. As $\mathbb{P}\left(\left\{\sigma_{n} \in \mathfrak{S}_{n, g}\right\} \cap\left\{\sigma_{n} \in \mathfrak{S}_{n} \backslash \mathfrak{S}_{n}^{p}\right\}\right)=0$, one can write $\mathbb{P}\left(\sigma_{n} \in \mathfrak{S}_{n, g}\right)=$ $\mathbb{P}\left(\sigma_{n} \in \mathfrak{S}_{n, g} \mid \sigma_{n} \in \mathfrak{S}_{n}^{p}\right) \mathbb{P}\left(\sigma_{n} \in \mathfrak{S}_{n}^{p}\right)$.

Using again the conjugation invariance, we obtain

$$
\mathbb{P}\left(\sigma_{n} \in \mathfrak{S}_{n, g} \mid \sigma_{n} \in \mathfrak{S}_{n}^{p}\right)=\frac{(n-2 p) !}{(n-p) !}
$$

and conclude the proof. 


\subsection{Proof of Proposition 1.3}

Proof. We will adapt the proof of [7, Lemma 14]. Let $e_{1} \geq 1$ be fixed. In the case $k=1$, since $C_{e_{1}}=1,(*)$ holds if we have:

$$
\begin{aligned}
& \forall \hat{g}_{1}, \hat{g}_{2} \in \widehat{\mathbb{G}}_{e_{1}}, \mathbb{P}\left(\left(\widehat{\mathcal{G}}_{1}^{1}\left(\sigma_{n}, \rho_{n}\right), \widehat{\mathcal{G}}_{2}^{1}\left(\sigma_{n}, \rho_{n}\right)\right)=\left(\hat{g}_{1}, \hat{g}_{2}\right)\right)=\frac{C_{\hat{g}_{1}, \hat{g}_{2}}}{n}+o\left(\frac{1}{n}\right) \\
& \text { and } \sum_{\hat{g}_{1}, \hat{g}_{2} \in \widehat{\mathbb{G}}_{e_{1}}} C_{\hat{g}_{1}, \hat{g}_{2}}=C_{e_{1}}=1 .
\end{aligned}
$$

Let $\hat{g}_{1}, \hat{g}_{2} \in \widehat{\mathbb{G}}_{e_{1}}$. We denote by

$$
p_{n}\left(\hat{g}_{1}, \hat{g}_{2}\right):=\mathbb{P}\left(\left(\widehat{\mathcal{G}}_{1}^{1}\left(\sigma_{n}, \rho_{n}\right), \widehat{\mathcal{G}}_{2}^{1}\left(\sigma_{n}, \rho_{n}\right)\right)=\left(\hat{g}_{1}, \hat{g}_{2}\right)\right) .
$$

Let $B_{\hat{g}_{1}, \hat{g}_{2}}^{n}$ be the set of couples $\left(g_{1}, g_{2}\right) \in\left(\mathbb{G}_{e_{1}}^{n}\right)^{2}$ having the same non-isolated vertices such that 1 is a non-isolated vertex of both graphs and, for $i \in\{1,2\}$, the equivalence class of $g_{i}$ is $\hat{g}_{i}$ and there exists $\sigma, \rho$ such that $\mathcal{G}_{1}^{1}(\sigma, \rho)=g_{1}$ and $\mathcal{G}_{2}^{1}(\sigma, \rho)=g_{2}$. By Lemma 2.4 and $H_{1}$, we have

$$
\begin{aligned}
p_{n}\left(\hat{g}_{1}, \hat{g}_{2}\right) & =\sum_{\left(g_{1}, g_{2}\right) \in B_{\hat{g}_{1}, \hat{g}_{2}}^{n}} \mathbb{P}\left(\left(\mathcal{G}_{1}^{1}\left(\sigma_{n}, \rho_{n}\right), \mathcal{G}_{2}^{1}\left(\sigma_{n}, \rho_{n}\right)\right)=\left(g_{1}, g_{2}\right)\right) \\
& =\sum_{\left(g_{1}, g_{2}\right) \in B_{\hat{g}_{1}, \hat{g}_{2}}^{n}} \mathbb{P}\left(\sigma_{n} \in \mathfrak{S}_{n, g_{1}}, \rho_{n} \in \mathfrak{S}_{n, g_{2}}\right) \\
& =\sum_{\left(g_{1}, g_{2}\right) \in B_{\hat{g}_{1}, \hat{g}_{2}}^{n}} \mathbb{P}\left(\sigma_{n} \in \mathfrak{S}_{n, g_{1}}\right) \mathbb{P}\left(\rho_{n} \in \mathfrak{S}_{n, g_{2}}\right)
\end{aligned}
$$

The graphs $\hat{g}_{1}, \hat{g}_{2}$ being fixed, it determines the number $v \leq 2 e_{1}$ of vertices of the graphs $g_{1}$ and $g_{2}$. In particular,

$$
\operatorname{card}\left(B_{\hat{g}_{1}, \hat{g}_{2}}^{n}\right) \leq\left(\begin{array}{c}
n-1 \\
v-1
\end{array}\right) v !^{2} .
$$

Indeed, there are $\left(\begin{array}{l}n-1 \\ v-1\end{array}\right)$ possible ways to choose the non-isolated vertices (other than 1) and for every choice of non isolated vertices, there is at most $v$ ! possible labellings of $\hat{g}_{1}$ (to obtain the graph $g_{1}$ ) and at most $v$ ! labellings $\hat{g}_{2}$ (to obtain the graph $g_{2}$ ). Let $p_{1}$ and $p_{2}$ be respectively the number of connected components of $g_{1}$ and $g_{2}$.

Starting from (2.3), we now distinguish different cases, depending on the structure of $\hat{g}_{1}$ and $\hat{g}_{2}$.

- Case 1: $\hat{g}_{1}$ and $\hat{g}_{2}$ have respectively $f_{1}$ and $f_{2}$ loops i.e edges of type $(i, i)$ with $f_{1}+f_{2}>0$. Then $2 p_{1}-f_{1} \leq v$ and $2 p_{2}-f_{2} \leq v$.

Consequently, by Lemmas 2.6 and 2.7,

$$
\begin{aligned}
p_{n}\left(\hat{g}_{1}, \hat{g}_{2}\right) & =o\left(n^{\frac{-f_{1}-f_{2}}{2}}\right) \sum_{\left(g_{1}, g_{2}\right) \in B_{\hat{g}_{1}, \hat{g}_{2}}^{n}} \frac{(n-v) !}{\left(n-p_{1}\right) !} \frac{(n-v) !}{\left(n-p_{2}\right) !} \\
& =\operatorname{card}\left(B_{\hat{g}_{1}, \hat{g}_{2}}^{n}\right) \frac{(n-v) !}{\left(n-p_{1}\right) !} \frac{(n-v) !}{\left(n-p_{2}\right) !} o\left(n^{\frac{-f_{1}-f_{2}}{2}}\right) \\
& \leq\left(\begin{array}{c}
n-1 \\
v-1
\end{array}\right) v !^{2} \frac{(n-v) !}{\left(n-p_{1}\right) !} \frac{(n-v) !}{\left(n-p_{2}\right) !} o\left(n^{\frac{-f_{1}-f_{2}}{2}}\right) \\
& =n^{v-1-\left(v-p_{1}+v-p_{2}\right)} o\left(n^{\frac{-f_{1}-f_{2}}{2}}\right)=o\left(n^{-1}\right) .
\end{aligned}
$$


A product of invariant random permutations has the same small cycle structure

- Case 2: $\hat{g}_{1}$ and $\hat{g}_{2}$ do not contain any loop, so that $p_{1} \leq \frac{v}{2}$ and $p_{2} \leq \frac{v}{2}$. Then, again by Lemma 2.6 ,

$$
\begin{aligned}
p_{n}\left(\hat{g}_{1}, \hat{g}_{2}\right) & \leq \sum_{\left(g_{1}, g_{2}\right) \in B_{\hat{g}_{1}, \hat{g}_{2}}^{n}} \frac{(n-v) !}{\left(n-p_{1}\right) !} \frac{(n-v) !}{\left(n-p_{2}\right) !} \\
& =\operatorname{card}\left(B_{\hat{g}_{1}, \hat{g}_{2}}^{n}\right) \frac{(n-v) !}{\left(n-p_{1}\right) !} \frac{(n-v) !}{\left(n-p_{2}\right) !} \\
& \leq\left(\begin{array}{l}
n-1 \\
v-1
\end{array}\right) v !^{2} \frac{(n-v) !}{\left(n-p_{1}\right) !} \frac{(n-v) !}{\left(n-p_{2}\right) !}=O\left(n^{v-1-\left(v-p_{1}+v-p_{2}\right)}\right) .
\end{aligned}
$$

Therefore, if $p_{1}<\frac{v}{2}$, as $p_{1} \leq \frac{v-1}{2}$ we have

$$
p_{n}\left(\hat{g}_{1}, \hat{g}_{2}\right)=O\left(n^{-\frac{3}{2}}\right) .
$$

The same holds if $p_{2}<\frac{v}{2}$ and the only remaining terms are the cases when $p_{1}=\frac{v}{2}=e_{1}$ and $p_{2}=\frac{v}{2}=e_{1}$. In this case, both graphs have necessarily connected components having two vertices. By Lemma 2.3 , we obtain that the only non trivial contribution comes from $\hat{g}_{1}=\hat{g}_{2}=\widehat{\mathcal{T}}_{e_{1}}$. By Lemma 2.9, we obtain

$$
\begin{aligned}
\left.\operatorname{card}\left(B_{\widehat{\mathcal{T}}_{e_{1}}, \widehat{\mathcal{T}}_{e_{1}}}^{n}\right) \frac{(n-v) !}{\left(n-p_{1}\right) !} \frac{(n-v) !}{\left(n-p_{2}\right) !}(1+o(1))\right) & \leq p_{n}\left(\widehat{\mathcal{T}}_{e_{1}}, \widehat{\mathcal{T}}_{e_{1}}\right) \\
& \leq \operatorname{card}\left(B_{\widehat{\mathcal{T}}_{e_{1}}, \widehat{\mathcal{T}}_{e_{1}}}^{n}\right) \frac{(n-v) !}{\left(n-p_{1}\right) !} \frac{(n-v) !}{\left(n-p_{2}\right) !} .
\end{aligned}
$$

Moreover, each element of $B_{\widetilde{\mathcal{T}}_{e_{1}}, \widehat{\tau}_{e_{1}}}^{n}$ can be characterized by a choice of $i_{2}^{1}, i_{3}^{1}, \ldots i_{e_{1}}^{1}$, $j_{1}^{1}, \ldots j_{e_{1}}^{1}$ pairwise distincts in $\{2,3, \ldots, n\}$, so that

$$
\operatorname{card}\left(B_{\widehat{\mathcal{T}}_{e_{1}}, \widehat{\mathcal{T}}_{e_{1}}}^{n}\right)=\frac{(n-1) !}{\left(n-2 e_{1}\right) !} .
$$

Since $v=2 p_{1}=2 p_{2}=2 e_{1}$, we get that

$$
p_{n}\left(\widehat{\mathcal{T}}_{e_{1}}, \widehat{\mathcal{T}}_{e_{1}}\right)=\frac{1+o(1)}{n} .
$$

Summarizing all cases, we get that $C_{\hat{g}_{1}, \hat{g}_{2}}=0$ unless $\hat{g}_{1}=\hat{g}_{2}=\widehat{\mathcal{T}}_{e_{1}}$, in which case $C_{\widehat{\mathcal{T}}_{e_{1}}, \widehat{\tau}_{e_{1}}}=1$.

\subsection{Proof of Theorem 1.2}

The proof of Theorem 1.2 is similar to that of Proposition 1.3. Instead of studying $\mathcal{G}_{i}^{1}$, we study $\mathcal{G}_{i}^{\{1,2, \ldots, k\}}$. We will prove using the same argument that only the event $\left\{(\sigma, \rho) ; \forall i \in\{1,2\}, \mathcal{G}_{i}^{\{1,2, \ldots, k\}}(\sigma, \rho) \in \cup_{p \geq 1} \mathcal{T}_{p}^{n}\right\}$ will contribute to the limit.

Proof of Theorem 1.2. Let $\mathbf{e}=\left(\mathbf{e}_{1}, \mathbf{e}_{2}, \ldots \mathbf{e}_{\mathbf{k}}\right)$ be fixed. Our goal is to compute $\mathbb{P}\left(\left(\sigma_{n}, \rho_{n}\right) \in\right.$ $\left.A_{\mathbf{e}}\right)$ where

$$
A_{\mathbf{e}}:=\left\{(\sigma, \rho) ; \forall i \leq k, c_{i}\left(\sigma^{-1} \rho\right)=e_{i}\right\} .
$$

If $\forall i \leq k, c_{i}\left(\sigma^{-1} \rho\right)=e_{i}$, then

$$
\widehat{\mathcal{G}}_{1}^{\{1,2, \ldots, k\}}(\sigma, \rho), \widehat{\mathcal{G}}_{2}^{\{1,2, \ldots, k\}}(\sigma, \rho) \in \bigcup_{p \leq 2 \sum_{i=1}^{k} e_{k}} \widehat{\mathbb{G}}_{p} .
$$


A product of invariant random permutations has the same small cycle structure

Since $\bigcup_{p \leq 2 \sum_{i=1}^{k} e_{k}} \widehat{\mathbb{G}}_{p}$ is finite and $A_{\mathbf{e}}$ can been as the disjoint union

$$
A_{\mathbf{e}}=\bigsqcup_{\hat{g}_{1}, \hat{g}_{2} \in \bigcup_{p \leq 2 \sum_{i=1}^{k} e_{k}} \widehat{\mathbb{G}}_{p}}\left(\left\{(\sigma, \rho) ;\left(\widehat{\mathcal{G}}_{1}^{\{1,2, \ldots, k\}}(\sigma, \rho), \widehat{\mathcal{G}}_{2}^{\{1,2, \ldots, k\}}(\sigma, \rho)\right)=\left(\hat{g}_{1}, \hat{g}_{2}\right)\right\} \cap A_{\mathbf{e}}\right)
$$

it is sufficient to prove that for any pair $\hat{g}_{1}, \hat{g}_{2} \in \bigcup_{p<2 \sum_{i=1}^{k} e_{k}} \widehat{\mathbb{G}}_{p}$ having the same number of non-isolated vertices, there exists a constant $C_{\hat{g}_{1}, \hat{g}_{2}, \mathrm{e}}$ such that under the assumptions of Theorem 1.2,

$$
\begin{aligned}
\mathbb{P}\left(\left(\sigma_{n}, \rho_{n}\right) \in\left\{(\sigma, \rho) ;\left(\widehat{\mathcal{G}}_{1}^{\{1,2, \ldots, k\}}(\sigma, \rho), \widehat{\mathcal{G}}_{2}^{\{1,2, \ldots, k\}}(\sigma, \rho)\right)\left(\hat{g}_{1}, \hat{g}_{2}\right)\right\}\right. & \left.\cap A_{\mathbf{e}}\right) \\
& =\frac{C_{\hat{g}_{1}, \hat{g}_{2}, \mathbf{e}}}{n^{k}}+o\left(\frac{1}{n^{k}}\right) .
\end{aligned}
$$

Let $\hat{g}_{1}, \hat{g}_{2} \in \bigcup_{p \leq 2 \sum_{i=1}^{k} e_{k}} \widehat{\mathbb{G}}_{p}$ be two unlabeled graphs having respectively $p_{1}$ and $p_{2}$ connected components and $v$ vertices. Let $B_{\hat{g}_{1}, \hat{g}_{2}}^{n, \mathbf{e}}$ be the set of couples $\left(g_{1}, g_{2}\right)$ with $n$ vertices, having the same non-isolated vertices such that

- $1,2, \ldots, k$ are non-isolated vertices of both graphs,

- for $i \in\{1,2\}$, the equivalence class of $g_{i}$ is $\hat{g}_{i}$,

- there exists $\sigma, \rho$ such that for $i \in\{1,2\}$ and $j \in\{1, \ldots, k\}, \mathcal{G}_{i}^{\{1,2, \ldots k\}}(\sigma, \rho)=g_{i}$ and $c_{j}\left(\sigma^{-1} \rho\right)=e_{j}$.

As before, we denote by

$$
p_{n, \mathbf{e}}\left(\hat{g}_{1}, \hat{g}_{2}\right):=\mathbb{P}\left(\left(\sigma_{n}, \rho_{n}\right) \in\left\{(\sigma, \rho) ;\left(\widehat{\mathcal{G}}_{1}^{\{1,2, \ldots, k\}}(\sigma, \rho), \widehat{\mathcal{G}}_{2}^{\{1,2, \ldots, k\}}(\sigma, \rho)\right)=\left(\hat{g}_{1}, \hat{g}_{2}\right)\right\} \cap A_{\mathbf{e}}\right)
$$

and we have

$$
\begin{aligned}
p_{n, \mathbf{e}}\left(\hat{g}_{1}, \hat{g}_{2}\right) & =\sum_{\left(g_{1}, g_{2}\right) \in B_{\hat{g}_{1}, \hat{g}_{2}}^{n, \mathbf{e}}} \mathbb{P}\left(\left(\mathcal{G}_{1}^{\{1,2, \ldots, k\}}\left(\sigma_{n}, \rho_{n}\right), \mathcal{G}_{2}^{\{1,2, \ldots, k\}}\left(\sigma_{n}, \rho_{n}\right)\right)=\left(g_{1}, g_{2}\right)\right) \\
& =\sum_{\left(g_{1}, g_{2}\right) \in B_{\hat{g}_{1}, \hat{g}_{2}}^{n, \mathbf{e}}} \mathbb{P}\left(\sigma_{n} \in \mathfrak{S}_{n, g_{1}}, \rho_{n} \in \mathfrak{S}_{n, g_{2}}\right) \\
& =\sum_{\left(g_{1}, g_{2}\right) \in B_{\hat{g}_{1}, \hat{g}_{2}}^{n, \mathbf{e}}} \mathbb{P}\left(\sigma_{n} \in \mathfrak{S}_{n, g_{1}}\right) \mathbb{P}\left(\rho_{n} \in \mathfrak{S}_{n, g_{2}}\right) .
\end{aligned}
$$

Starting from there, we distinguish different cases:

- Case 1: $\hat{g}_{1}$ and $\hat{g}_{2}$ have respectively $f_{1}$ and $f_{2}$ loops i.e edges of type $(i, i)$ with $f_{1}+f_{2}>0$. Then $2 p_{1}-f_{1} \leq v$ and $2 p_{2}-f_{2} \leq v$. Consequently, by Lemmas 2.6 and 2.7,

$$
\begin{aligned}
p_{n, \mathbf{e}}\left(\hat{g}_{1}, \hat{g}_{2}\right) & =\operatorname{card}\left(B_{\hat{g}_{1}, \hat{g}_{2}}^{n, \mathbf{e}}\right) \frac{(n-v) !}{\left(n-p_{1}\right) !} \frac{(n-v) !}{\left(n-p_{2}\right) !} o\left(n^{\frac{-f_{1}-f_{2}}{2}}\right) \\
& \leq\left(\begin{array}{c}
n-k \\
v-k
\end{array}\right) v !^{2} \frac{(n-v) !}{\left(n-p_{1}\right) !} \frac{(n-v) !}{\left(n-p_{2}\right) !} o\left(n^{\frac{-f_{1}-f_{2}}{2}}\right) \\
& =n^{v-k-\left(v-p_{1}+v-p_{2}\right)} o\left(n^{\frac{-f_{1}-f_{2}}{2}}\right)=o\left(n^{-k}\right) .
\end{aligned}
$$

- Case 2: $\hat{g}_{1}$ and $\hat{g}_{2}$ do not contain any loop. Then $p_{1} \leq \frac{v}{2}$ and $p_{2} \leq \frac{v}{2}$. Consequently,

$$
\begin{aligned}
p_{n, \mathbf{e}}\left(\hat{g}_{1}, \hat{g}_{2}\right) & \leq \operatorname{card}\left(B_{\hat{g}_{1}, \hat{g}_{2}}^{n, \mathbf{e}}\right) \frac{(n-v) !}{\left(n-p_{1}\right) !} \frac{(n-v) !}{\left(n-p_{2}\right) !} \\
& \leq\left(\begin{array}{c}
n-k \\
v-k
\end{array}\right) v !^{2} \frac{(n-v) !}{\left(n-p_{1}\right) !} \frac{(n-v) !}{\left(n-p_{2}\right) !} \leq C n^{v-k-\left(v-p_{1}+v-p_{2}\right)}
\end{aligned}
$$


A product of invariant random permutations has the same small cycle structure

Therefore, if $p_{1}<\frac{v}{2}$ or $p_{2}<\frac{v}{2}$ then $p_{n, \mathbf{e}}\left(\hat{g}_{1}, \hat{g}_{2}\right)=o\left(n^{-k}\right)$. The only remaining terms are the cases when $p_{1}=\frac{v}{2}$ and $p_{2}=\frac{v}{2}$. In this case, both graphs have necessarily only connected components having two vertices. Assume that one of the two graphs has a cycle. Then, by Lemma 2.8, we have

$$
\begin{aligned}
p_{n, \mathbf{e}}\left(\hat{g}_{1}, \hat{g}_{2}\right) & \leq \sum_{\left(g_{1}, g_{2}\right) \in B_{\hat{g}_{1}, \hat{g}_{2}}^{n, \mathbf{g}}}\left(\mathbb{P}\left(c_{1}\left(\sigma_{n}\right)=2\right)+\mathbb{P}\left(c_{1}\left(\rho_{n}\right)=2\right)\right) \frac{(n-v) !}{\left(n-p_{1}\right) !} \frac{(n-v) !}{\left(n-p_{2}\right) !} \\
& \leq C\left(\mathbb{P}\left(c_{1}\left(\sigma_{n}\right)=2\right)+\mathbb{P}\left(c_{1}\left(\rho_{n}\right)=2\right)\right) n^{-k} .
\end{aligned}
$$

Under $H_{4}$, we have $\left.\mathbb{P}\left(c_{1}\left(\sigma_{n}\right)=2\right)+\mathbb{P}\left(c_{1}\left(\rho_{n}\right)=2\right)\right)=o(1)$ so that $p_{n, \mathbf{e}}\left(\hat{g}_{1}, \hat{g}_{2}\right)=$ $o\left(n^{-k}\right)$ as soon as one of the graph has a cycle.

As before, the only non-trivial contributions come from the cases when $\hat{g}_{1}=\hat{g}_{2}=\widehat{\mathcal{T}}_{p}$ for some $p \leq \sum_{i=1}^{k} e_{i}$ and by Lemma 2.9, we obtain

$$
\begin{aligned}
\operatorname{card}\left(B_{\widehat{\mathcal{T}}_{p}, \widehat{\mathcal{T}}_{p}}^{n, \mathbf{T}}\right) \frac{(n-v) !}{\left(n-p_{1}\right) !} \frac{(n-v) !}{\left(n-p_{2}\right) !}\left(1-O\left(\frac{1}{n}\right)\right) & \leq p_{n, \mathbf{e}}\left(\widehat{\mathcal{T}}_{p}, \widehat{\mathcal{T}}_{p}\right) \\
& \leq \operatorname{card}\left(B_{\widehat{\mathcal{T}}_{p}, \widehat{\tau}_{p}}^{n, \mathbf{e}}\right) \frac{(n-v) !}{\left(n-p_{1}\right) !} \frac{(n-v) !}{\left(n-p_{2}\right) !} .
\end{aligned}
$$

One can conclude since, for any $n \geq 2 p$,

$$
\operatorname{card}\left(B_{\widehat{\mathcal{T}}_{p}, \widehat{,}_{p}}^{n, \mathbf{e}}\right)=\operatorname{card}\left(B_{\widehat{\mathcal{T}}_{p}, \widehat{\mathcal{T}}_{p}}^{2 p, \mathbf{e}}\right)\left(\begin{array}{c}
n-k \\
2 p-k
\end{array}\right)
$$

Indeed, one can write $B_{\widehat{\mathcal{T}}_{p}, \widehat{\mathcal{T}}_{p}}^{n, \mathbf{e}}$ as the disjoint union

$$
\bigcup_{k+1 \leq \ell_{1}<\ldots<\ell_{2 p-k} \leq n}\left(B_{\widetilde{\mathcal{T}}_{p}, \widehat{T}_{p}}^{n, \mathbf{e}} \cap\left\{1, \ldots, k, \ell_{1}, \ldots, \ell_{2 p-k} \text { are the non-isolated vertices }\right\}\right)
$$

where each set has the same cardinal, which is equal to $\operatorname{card}\left(B_{\widetilde{\tau}_{p}, \widehat{\tau}_{p}}^{2 p, \mathbf{e}}\right)$.

Consequently, $C_{\hat{g}_{1}, \hat{g}_{2}, \mathbf{e}}=0$, unless there exists $p \leq \sum_{i=1}^{k} e_{i}$ such that $\left(\hat{g}_{1}, \hat{g}_{2}\right)=\left(\widehat{\mathcal{T}}_{p}, \widehat{\mathcal{T}}_{p}\right)$ and then

$$
C_{\widehat{\mathcal{T}}_{p}, \widehat{\tau}_{p}, \mathbf{e}}=\frac{\operatorname{card}\left(B_{\widehat{\mathcal{T}_{p}}, \widehat{\mathcal{T}_{p}}}^{2 p, \mathbf{e}}\right)}{(2 p-k) !} .
$$

As the constants $C_{\hat{g}_{1}, \hat{g}_{2}, \mathrm{e}}$ do not depend on the distributions of $\sigma_{n}$ and $\rho_{n}$, this concludes the proof of Theorem 1.2.

\section{Further discussion}

\subsection{Extension to the product of more than two permutations}

Using the same technique of proof, one can obtain a similar result for the product of $m$ permutations.

Proposition 3.1. Let $m \geq 2$. For $1 \leq \ell \leq m$, let $\left(\sigma_{n}^{\ell}\right)_{n \geq 1}$ be a sequence of random permutations such that for any $n \geq 1, \sigma_{n}^{\ell} \in \mathfrak{S}_{n}$. For any $k \geq 1$, let $t_{k}^{n}:=\#_{k}\left(\prod_{\ell=1}^{m} \sigma_{n}^{\ell}\right)$. Assume that

- For any $n \geq 1,\left(\sigma_{n}^{1}, \ldots, \sigma_{n}^{\ell}\right)$ are independent.

- For any $n \geq 1$ and $1 \leq \ell \leq m$, for any $\sigma \in \mathfrak{S}_{n}$,

$$
\sigma^{-1} \sigma_{n}^{\ell} \sigma \stackrel{d}{=} \sigma_{n}^{\ell}
$$

except maybe for one $\ell \in\{1, \ldots, m\}$. 
A product of invariant random permutations has the same small cycle structure

- There exist $1 \leq i<j \leq m$ such that for any $k \geq 1$,

$$
\begin{gathered}
\lim _{n \rightarrow \infty} \mathbb{E}\left(\left(\frac{\#_{1} \sigma_{n}^{i}}{\sqrt{n}}\right)^{k}\right)=0 \quad \text { and } \lim _{n \rightarrow \infty} \mathbb{E}\left(\left(\frac{\#_{1} \sigma_{n}^{j}}{\sqrt{n}}\right)^{k}\right)=0, \\
\lim _{n \rightarrow \infty} \frac{\mathbb{E}\left(\#_{2} \sigma_{n}^{i}\right)}{n}=0 \quad \text { and } \quad \lim _{n \rightarrow \infty} \frac{\mathbb{E}\left(\#_{2} \sigma_{n}^{j}\right)}{n}=0 .
\end{gathered}
$$

Then for any $k \geq 1$,

$$
\left(t_{1}^{n}, t_{2}^{n}, \ldots, t_{k}^{n}\right) \underset{n \rightarrow \infty}{\stackrel{d}{\longrightarrow}} \eta_{k} .
$$

The case $m=2$ is given is Theorem 1.2. To extend to $m>2$, we will proceed by induction on the number $m$ of permutations using the following lemma.

Lemma 3.2. Let $\left(\sigma_{n}^{1}\right)_{n \geq 1},\left(\sigma_{n}^{2}\right)_{n \geq 1}$ be two sequences of random permutations such that for any $n \geq 1, \sigma_{n}^{1}, \sigma_{n}^{2} \in \mathfrak{S}_{n}$. Assume that $\left(H_{1}\right)$ and $\left(H_{2}\right)$ hold and that, for any $k \geq 1$,

$$
\lim _{n \rightarrow \infty} \mathbb{E}\left(\left(\frac{\#_{1} \sigma_{n}^{1}}{\sqrt{n}}\right)^{k}\right)=0 \quad \text { and } \quad \lim _{n \rightarrow \infty} \frac{\mathbb{E}\left(\#_{2} \sigma_{n}^{1}\right)}{n}=0,
$$

then

$$
\lim _{n \rightarrow \infty} \mathbb{E}\left(\left(\frac{\#_{1}\left(\sigma_{n}^{1} \sigma_{n}^{2}\right)}{\sqrt{n}}\right)^{k}\right)=0 \text { and } \lim _{n \rightarrow \infty} \frac{\mathbb{E}\left(\#_{2}\left(\sigma_{n}^{1} \sigma_{n}^{2}\right)\right)}{n}=0 .
$$

Proof. We will only give a sketch of the proof. The idea is to repeat the same study as in the case $m=2$ in the two particular quantities.

- Take $k \geq 1$ and $e_{1}=e_{2}=\cdots=e_{k}=1$. One can show that, under the hypotheses of Lemma 3.2,

$$
\lim _{n \rightarrow \infty} \sum_{\hat{g}_{i}, \hat{g}_{i}^{\prime} \in \widehat{\mathbb{G}}_{1}, 1 \leq i \leq k} n^{\frac{k}{2}} \mathbb{P}\left(\widehat{\mathcal{G}}^{\{1,2, \ldots, k\}}\left(\sigma_{n}^{1}, \sigma_{n}^{2}\right)=\left(\hat{g}_{1}, \hat{g}_{1}^{\prime}, \hat{g}_{2}, \ldots \hat{g}_{k}^{\prime}\right)\right)=0 .
$$

This leads to the first limit in (3.1).

- Take $k=1$ and $e_{1}=2$. One can show that, under the hypotheses of Lemma 3.2,

$$
\lim _{n \rightarrow \infty} \sum_{\hat{g}_{1}, \hat{g}_{2} \in \widehat{\mathbb{G}}_{2},} \mathbb{P}\left(\left(\widehat{\mathcal{G}}_{1}^{1}\left(\sigma_{n}^{1}, \sigma_{n}^{2}\right), \widehat{\mathcal{G}}_{2}^{1}\left(\sigma_{n}^{1}, \sigma_{n}^{2}\right)\right)=\left(\hat{g}_{1}, \hat{g}_{2}\right)\right)=0 .
$$

This leads to the second limit in (3.1).

\subsection{Optimality}

In this last subsection, we make a few remarks on the optimality of the assumptions $H_{3}$ and $H_{4}$ in Theorem 1.2. We assume hereafter that $H_{1}$ and $H_{2}$ hold true and consider for the sake of clarity the case $m=2$.

- The assumption $H_{3}$ is optimal in the sense that

$$
\begin{aligned}
& \text { if } \liminf _{n \rightarrow \infty} n^{-\frac{k}{2}} \min \left(\mathbb{E}\left(\left(\#_{1} \sigma_{n}\right)^{k}\right), \mathbb{E}\left(\left(\#_{1} \rho_{n}\right)^{k}\right)\right)=\varepsilon_{k}>0, \\
& \text { then } \liminf _{n \rightarrow \infty} \mathbb{E}\left(\left(\#_{1}\left(\sigma_{n} \rho_{n}\right)\right)^{k}\right) \geq \mathbb{E}\left(\xi_{1}^{k}\right)+\varepsilon_{k}^{2} .
\end{aligned}
$$

Indeed, going back to the equation $(*)$, one can see that in the case $e_{1}=e_{2}=$ $\cdots=e_{k}=1$, if $\hat{g}$ is the class of the graph with adjacency matrix $\operatorname{Id}_{k}$ the event $\left\{\left(\widehat{\mathcal{G}}_{1}^{1,2, \ldots, k}\left(\sigma_{n}, \rho_{n}\right), \widehat{\mathcal{G}}_{2}^{1,2, \ldots, k}\left(\sigma_{n}, \rho_{n}\right)\right)=(\hat{g}, \hat{g})\right\}$ will contribute to the limit, leading to the term $\varepsilon_{k}^{2}$. 
A product of invariant random permutations has the same small cycle structure

- Similarly $H_{4}$ is optimal in the sense that if $\liminf _{n \rightarrow \infty}\left(\frac{\min \left(\mathbb{E}\left(\#_{2} \sigma_{n}\right), \mathbb{E}\left(\#_{2} \rho_{n}\right)\right)}{n}\right)=\varepsilon^{\prime}>0$, then $\liminf _{n \rightarrow \infty} \mathbb{E}\left(\left(\#_{1}\left(\sigma_{n} \rho_{n}\right)\right)^{2}\right) \geq 2+\varepsilon^{\prime 2}$

Indeed, as above, in the case $e_{1}=e_{2}=1$, if $\hat{g}^{\prime}$ is the class of the graph with adjacency matrix $\left(\begin{array}{ll}0 & 1 \\ 1 & 0\end{array}\right)$, the event $\left\{\left(\widehat{\mathcal{G}}_{1}^{1,2, \ldots, k}\left(\sigma_{n}, \rho_{n}\right), \widehat{\mathcal{G}}_{2}^{1,2, \ldots, k}\left(\sigma_{n}, \rho_{n}\right)\right)=\left(\hat{g}^{\prime}, \hat{g}^{\prime}\right)\right\}$ will contribute to the limit.

- Assume now that one of the bounds in $H_{3}$ is not satisfied. More precisely, assume that there exists $k \geq 1$ such that

$$
\liminf _{n \rightarrow \infty} n^{-\frac{k}{2}} \mathbb{E}\left(\left(\#_{1} \sigma_{n}\right)^{k}\right)=\varepsilon_{k}>0 \text {, or } \liminf _{n \rightarrow \infty} \frac{\mathbb{E}\left(\#_{2} \sigma_{n}\right)}{n}=\varepsilon^{\prime}>0 .
$$

Then, by similar arguments, one can check that the convergences

$$
\forall k \geq 1, \lim _{n \rightarrow \infty} n^{-\frac{k}{2}} \mathbb{E}\left(\left(\#_{1} \rho_{n}\right)^{k}\right)=0 \quad \text { and } \quad \lim _{n \rightarrow \infty} \frac{\mathbb{E}\left(\#_{2} \rho_{n}\right)}{n}=0
$$

are a necessary condition to obtain (1.1) and that the convergences

$$
\forall k \geq 1, \lim _{n \rightarrow \infty} n^{-\frac{k}{2}} \mathbb{E}\left(\left(\#_{1} \rho_{n}\right)^{k}\right)=0, \limsup _{n \rightarrow \infty} n^{-\frac{k}{2}} \mathbb{E}\left(\left(\#_{1} \sigma_{n}\right)^{k}\right)<\infty
$$

and $\quad \lim _{n \rightarrow \infty} \frac{\mathbb{E}\left(\#_{2} \rho_{n}\right)}{n}=0$ are a sufficient condition to obtain (1.1).

\section{References}

[1] Richard Arratia, Andrew D. Barbour, and Simon Tavaré, Limits of logarithmic combinatorial structures, Ann. Probab. 28 (2000), no. 4, 1620-1644. MR-1813836

[2] Richard Arratia, Andrew D. Barbour, and Simon Tavaré Logarithmic combinatorial structures: A probabilistic approach (Ems monographs in mathematics), European Mathematical Society, 2003. MR-2032426

[3] Thomas Budzinski, Nicolas Curien, and Bram Petri, Universality for random surfaces in unconstrained genus, Electron. J. Combin. 26 (2019), no. 4, Paper 4.2, 34. MR-4025406

[4] Sergei Chmutov and Boris Pittel, On a surface formed by randomly gluing together polygonal discs, Adv. in Appl. Math. 73 (2016), 23-42. MR-3433499

[5] Alex Gamburd, Poisson-Dirichlet distribution for random Belyi surfaces, Ann. Probab. 34 (2006), no. 5, 1827-1848. MR-2271484

[6] Mohamed Slim Kammoun, Monotonous subsequences and the descent process of invariant random permutations, Electron. J. Probab. 23 (2018), Paper no. 118, 31. MR-3885551

[7] Mohamed Slim Kammoun, On the longest common subsequence of independent random permutations invariant under conjugation, arXiv e-prints (2019), arXiv:1904.00725.

[8] Camille Male, Traffic distributions and independence: permutation invariant random matrices and the three notions of independence, arXiv e-prints (2011), arXiv:1111.4662.

[9] Sumit Mukherjee, Fixed points and cycle structure of random permutations, Electron. J. Probab. 21 (2016), Paper No. 40, 18. MR-3515570

Acknowledgments. The first author would like to acknowledge a useful discussion with Camille Male about traffic distributions and both authors are grateful to Valentin Féray for pointing relevant references. We also thank an anonymous referee for the careful reading of the paper and for their help in the proof that $\left(^{*}\right)$ implies Theorem 1.2. 